\title{
Stochastic higher order finite element elasto-plastic analysis of the necking phenomenon
}

\author{
Michat Strąkowski ${ }^{1, *}$, and Marcin Kamiński ${ }^{1}$ \\ ${ }^{1}$ Łódź University of Technology, Faculty of Civil Engineering, Architecture and Environmental Engineering, Department of \\ Structural Mechanics, Politechniki Avenue 6, 90-924 Łódź, Poland
}

\begin{abstract}
The principal goal of this work is to investigate an application of the stochastic perturbation technique of the $10^{\text {th }}$ order in coupled thermo-elasto-plastic analysis of tension of the steel elastic bar exposed to fire with thermally dependent material characteristics. An ambient temperature, calculated from the fire curve after ISO 834-1, equivalent to the fire exposure of the steel structure is treated here as the input Gaussian random variable. It is uniquely defined by the constant mean value at outer surfaces of this element, where material parameters of the steel as Young modulus, yield strength, heat conductivity, capacity and thermal elongation are considered all as highly temperature-dependent. Computational implementation known as the Stochastic Finite Element Method is carried out with the use of the FEM system ABAQUS and computer algebra system MAPLE. It uses both polynomial and non-polynomial local response functions of stresses and displacements. The basic probabilistic characteristics of time-dependent structural response are determined (expectations, coefficients of variation, skewness and kurtosis) and verified with classical Monte-Carlo simulation scheme and semi-analytical technique for input coefficient of variation not larger than 0.20 . Finally, probabilistic convergence of all three methods versus increasing input uncertainty level is investigated.
\end{abstract}

\section{Introduction}

The influence of a fire on the behaviour of steel structures is always very interesting. It is clear that the total time from a fire ignition up to its failure strongly depends on the basic structural materials as well as on the protection cover systems. The determination of this influence is very challenging. There are a number of simulation techniques available like fuzzy sets theory, analytical methods, and others based on Taylor expansions methods known as perturbation techniques. In this paper, we use the well-known Monte Carlo method, semi-analytical method and $10^{\text {th }}$ order perturbation method implemented with the FEM originating from the Second Order Second Moment method [1]. We compute four probabilistic coefficients to gain more information about probability density function itself. The basic probabilistic moments, as well as coefficients like skewness and kurtosis, are calculated by full symbolic expansion of integral definitions and partial differentiation of the nodal response functions with respect of gas temperature, which is a random input variable. In this paper, we consider strength test of elasto-plastic steel cylindrical specimens subjected to fire known from literature. In this case, all physical and mechanical material properties like Young modulus, yield strength, conductivity, heat capacity and linear, thermal elongation are temperature-dependent. Gas temperatures are taken from ISO-834-1 [2]. It is the main load in this paper.

\section{Theoretical background}

We use the random variable $b$ and its probability density function $p_{b}(x)$. The first probabilistic moment of this variable is defined as [3]

$$
E[b] \equiv b^{0}=\int_{-\infty}^{+\infty} b p_{b}(x) d x,
$$

where $b^{0}$ means the average value of the $b$. Based on Eq. (1) we determine variance of $b$ as follows

$$
\operatorname{Var}[b]=\int_{-\infty}^{+\infty}[b-E(b)]^{2} p_{b}(x) d x .
$$

The third and fourth central probabilistic moments and related coefficients may be defined based on the classical definitions from the probability theory. The basic idea of the stochastic perturbation approach is to expand all the resulting state functions, and all input random variables of the given boundary initial problems via Taylor series about their expectations using the perturbation parameter marked $\varepsilon$. The random state

\footnotetext{
Corresponding author: michal.strakowski@p.lodz.pl
} 
function $f$ referred to its parameter $b$ around its mean value is given as follows

$$
f=f^{0}+\sum_{n=10}^{1} \frac{\varepsilon^{n}}{n !} \frac{\partial^{n} f}{\partial b^{n}} \Delta b^{n},
$$

where

$$
\varepsilon \Delta b=\varepsilon\left(b-b^{0}\right),
$$

is the first variation of variable $b$ about its expected value. Further, we analyze the expected values of displacements $u(b)$ defined analogously to the (3) by its expansion via Tylor series as follows

$$
\begin{aligned}
& E[u(b)]=\int_{-\infty}^{+\infty} u(b) p_{b}(x) d x= \\
& =\int_{-\infty}^{+\infty}\left(u^{0}\left(b^{0}\right)+\sum_{n=10}^{1} \frac{\varepsilon^{n}}{n !} \frac{\partial^{n} u(b)}{\partial b^{n}} \Delta b^{n}\right) p_{b}(x) d x
\end{aligned} .
$$

Notice that this power expansion is valid only if the state function is analytic and the all series converge. Therefore, any criteria of convergence should include the magnitude of the perturbation parameter which is taken as equal $\varepsilon=1$ in several practical computations and here also [4-5]. It brings for the input random variable with symmetric probability density function in the second order perturbation approach

$$
\begin{aligned}
& E[u(b)]=u^{0}\left(b^{0}\right)+\frac{1}{2 !} \frac{\partial^{2} u(b)}{\partial b^{2}} \mu_{2}(b)+\cdots+ \\
& +\frac{1}{10 !} \frac{\partial^{10} u(b)}{\partial b^{10}} \mu_{10}(b)
\end{aligned},
$$

where $\mu_{n}(b)$ denotes $n$th order the central probabilistic moment of the variable $b$. We use $10^{\text {th }}$ order approach so the orders higher than the $10^{\text {th }}$ are neglected. Variance of the displacements state function approach as

$$
\operatorname{Var}[u(b)]=\mu_{2}(u(b))=\int_{-\infty}^{+\infty}(u(b)-E[u(b)])^{2} p_{b}(x) d x
$$

using the $10^{\text {th }}$ order approach we define the $3^{\text {rd }}$ central probabilistic moment as

$$
\mu_{3}(u(b))=\int_{-\infty}^{+\infty}(u(b)-E[u(b)])^{3} p_{b}(x) d x,
$$

and the $4^{\text {th }}$ central probabilistic moment may be given as

$$
\mu_{4}(u(b))=\int_{-\infty}^{+\infty}(u(b)-E[u(b)])^{4} p_{b}(x) d x .
$$

Based on classical definition of the variance we can calculate the coefficient of variation as follows

$$
\alpha(u(b))=\frac{\sqrt{\operatorname{Var}[u(b)]}}{E[u(b)]} .
$$

We compute skewness and kurtosis to recognize the type of probabilistic distribution of the state function. Skewness is a ratio of the third central probabilistic moment and the third power of standard deviation as

$$
S(u(b))=\frac{\mu_{3}(u(b))}{[\sqrt{\operatorname{Var}[u(b)]}]^{3}},
$$

and kurtosis is given as

$$
\kappa(u(b))=\frac{\mu_{4}(u(b))}{[\sqrt{\operatorname{Var}[u(b)}]]^{4}}-3 .
$$

Let us mention that, at this stage, the proposed procedure is independent from a choice of the initial probability distribution function. However, a satisfactory probabilistic convergence of the final results may demand various lengths of the expansions for random variables with different distributions. In these computations, we assumed Gaussian probability distribution for this function.

The approach is based on the iterative fully-coupled deterministic FEM [6] equations as follows

$$
\left\{\begin{array}{l}
\mathbf{C}^{(\alpha)} \dot{\boldsymbol{\theta}}^{(\alpha)}+\hat{\mathbf{K}}^{(\alpha)} \boldsymbol{\theta}^{(\alpha)}=\hat{\mathbf{R}}^{(\alpha)} \\
\mathbf{K}^{(\alpha)}(\boldsymbol{\theta}) \mathbf{q}^{(\alpha)}(\boldsymbol{\theta})=\mathbf{R}^{(\alpha)}
\end{array},\right.
$$

where $\mathbf{C}$ is heat capacity matrix, $\boldsymbol{\theta}$ is nodal temperatures vector, $\hat{\mathbf{K}}$ is heat conductivity matrix, $\mathbf{K}$ denotes stiffness matrix, $\mathbf{R}$ is matrix of nodal loads, $\hat{\mathbf{R}}$ is a matrix of thermal loads and $\mathbf{q}$ is the solution displacement vector and $\alpha$ indices the test number relevant to Response Function Method [7]. We solve Eq. (13) in ABAQUS system eleven times because we assume dispersion of the random variable in range $b=\left[b^{0}-\Delta b ; b^{0}+\Delta b\right]$, where $2 \Delta b=0,05 b$. Further determination of the probabilistic moments from Eqs. $(5 \div 12)$ thanks to the symbolic derivation of all partial derivatives with respect to the given random variable $b$. We carry out all these calculations in computer algebra system MAPLE.

\section{Numerical simulation}

The numerical example consists of the necking of an axisymmetric steel specimen phenomenon [8]. The initial length of the bar is $2 L_{0}$ and the initial radius is $R_{0}$. We assume that $L_{0} / R_{0}=4$. We take a small cut of its right bottom corner $\Delta R=0.005 R_{0} \times 0.05 \mathrm{~mm}$ to ensure necking in the middle of it (Fig. 1). 


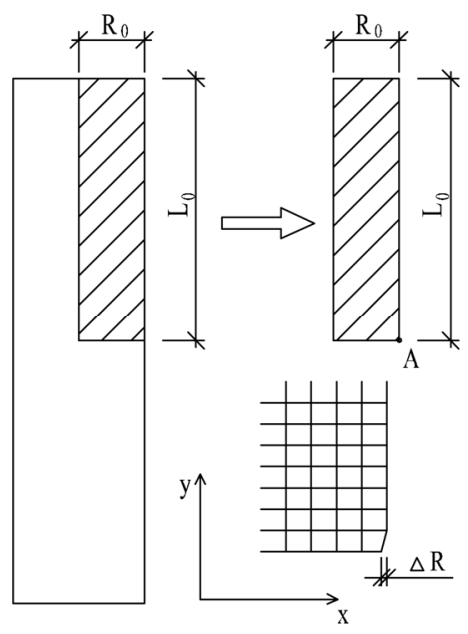

Fig. 1. Geometry and imperfection of the specimen.

4-noded FEM elements [9] are used for discretization and divide the specimen into 1600 pieces signed in ABAQUS system - CAX4RT. It means that we use 4noded transient heat transfer elements with reduced integration. Each node has 7 degrees of freedom (6 mechanicals and 1 thermal which is temperature). Mechanical boundary conditions are $u_{x}=0$ on the left side, $u_{y}=0$ on the bottom side and forced displacement $u_{y}=0.125 L_{0}$ applied to the top side (Fig. 2). In the beginning temperatures are set equal to $T_{0}=20^{\circ} \mathrm{C}$ for all nodal points, while fire simulation is performed by setting surface temperatures equal to these calculated from the fire curve after ISO 834-1 [2] statement. We apply these temperatures to the top and the right side of the sample (Fig. 2).
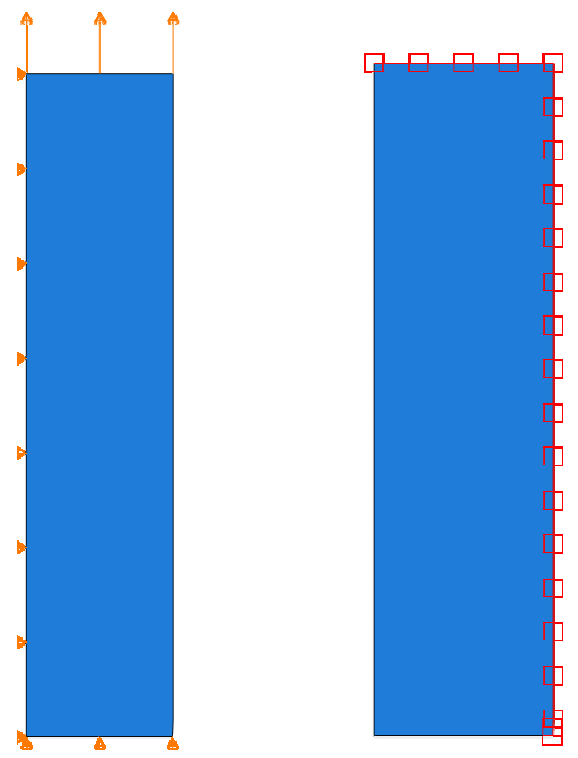

Fig. 2. Mechanical (left) and thermal (right) boundary conditions.

Steel properties as Young modulus, conductivity, yield strength, specific heat and coefficient of elongation are highly temperature-dependent based on EN 1993-1-2 [10]. We use fully coupled thermal-stress analysis because we look at the phenomenon where element temperatures affect stress and displacement values. 11 tests were carried out with the time period equal to 900 seconds for 11 different values of the gas temperatures. It is a random variable $b \equiv T_{s}$ in this case.

Figure 3 shows the resulting displacement distribution on deformed specimens at the moment $t=300,900 \mathrm{~s}$. We can see the difference between the length of it at the beginning and the end of the experiment. Necking is visible as well.
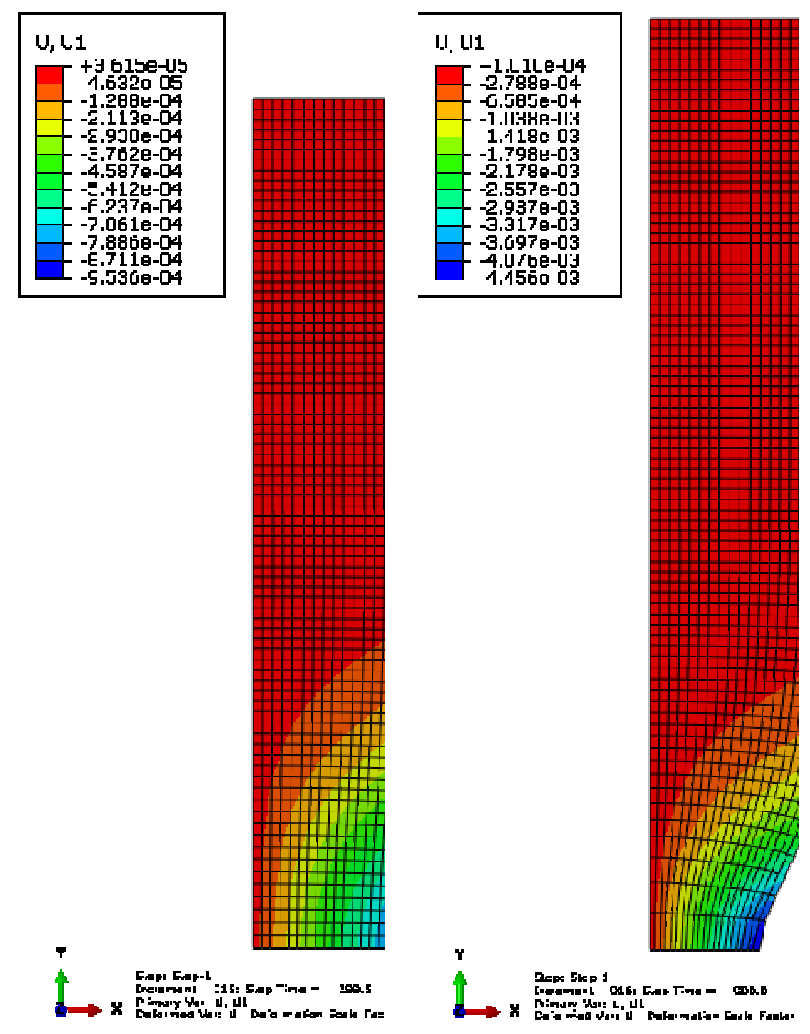

Fig. 3. Displacement at $t=300,900 \mathrm{~s}$.

To describe the response of the structure we determined 18 different polynomial functions based on results from the FEM tests (Fig. 4).

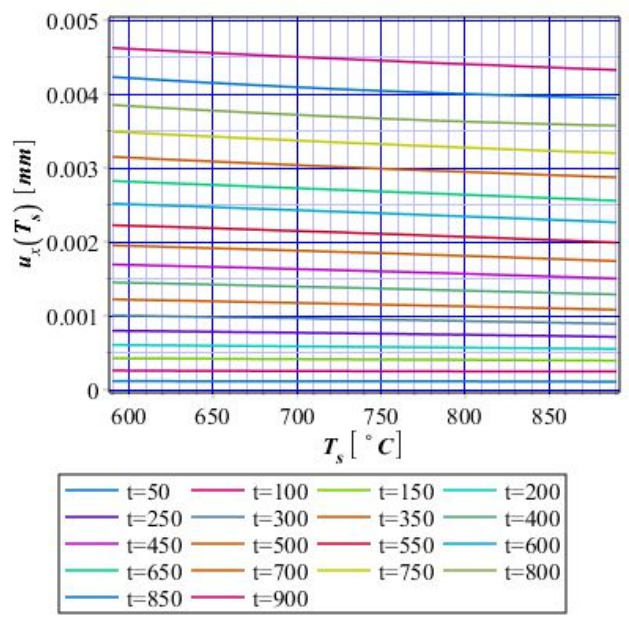

Fig. 4. Displacements response functions. 
We are reminded that gas temperature is a random variable in this case with its input coefficient of variation $\alpha\left(T_{s}\right)$ in the range of $[0.0,0.2]$. Figures $5 \div 8$ show expectations, coefficients of variation, skewness and kurtosis for extreme horizontal displacements in point $A$ (Fig. 1) of the specimen as a time-dependent function. The expectations of displacements are concentrated around its mean values. We can observe very good convergence of the results obtained from semi-analytical (AM), Monte Carlo Simulation (MCS) and perturbation technique (PM). Expectations (Fig. 5) are independent of the input coefficient of variation $\alpha\left(T_{s}\right)$.

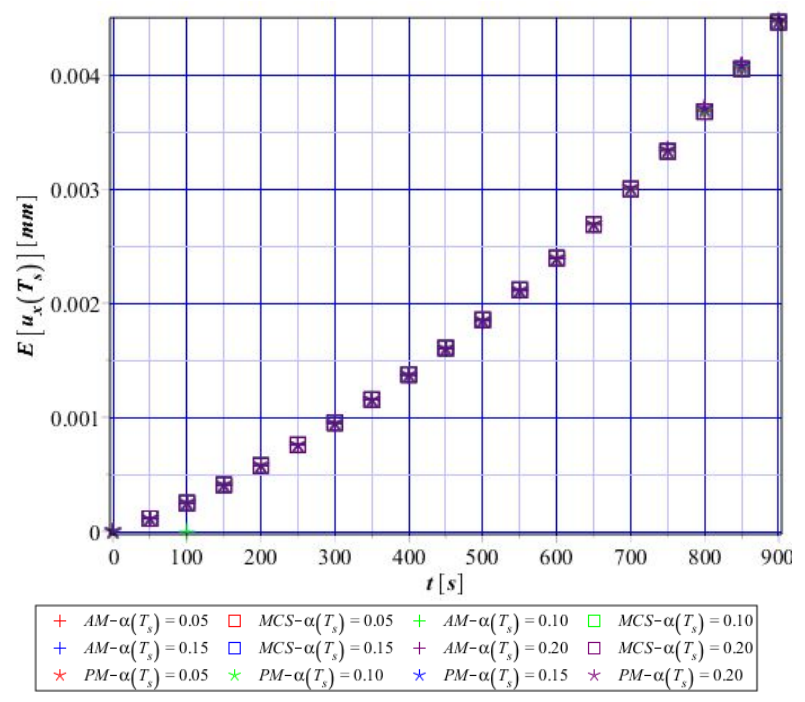

Fig. 5. Expectations of displacements.

Coefficients of variation (Fig. 6) are close to zero (small dispersion) for Monte Carlo Simulation (MCS) and it means that values of displacements are concentrated round its means. Using perturbation method (PM) and semi-analytical method (AM) for these calculations we obtain smaller values of the output than the input coefficients of variation in entire range $\alpha\left(T_{s}\right) \in[0 ; 0.20]$.

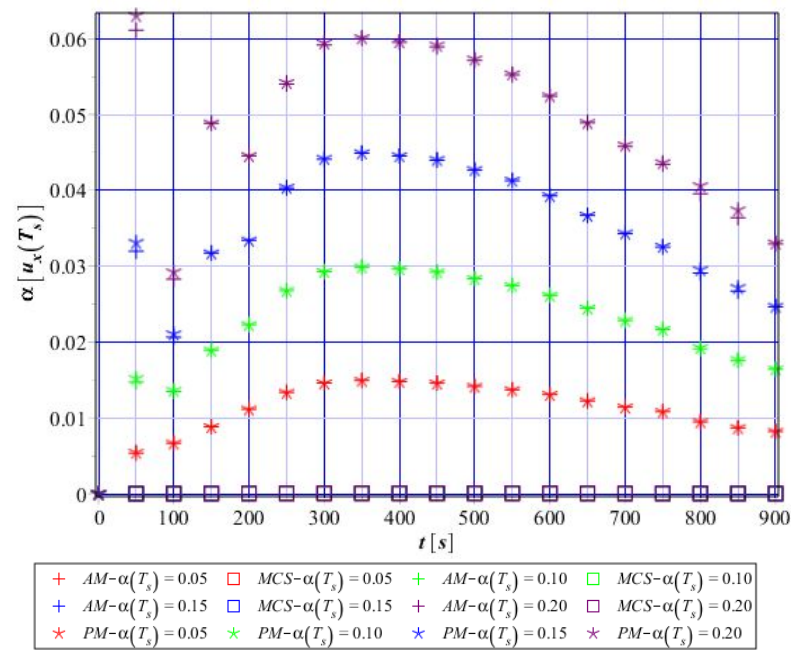

Fig. 6. Coefficient of variation of displacements.
Studying skewness distributions (Fig. 7) we can see fluctuations at the beginning and at the end of the analysis. According to this plot we can conclude that horizontal displacements $\mathrm{u}_{\mathrm{x}}$ distribution is Gaussian when $\alpha\left(T_{s}\right) \leq 0.05$ because in this range skewness is close to zero based on all three approaches. Using Monte Carlo Simulation (MCS) we obtain smaller values in comparison with other methods. During all the time, skewness has positive and negative values, which brings problems with recognition of its distribution.

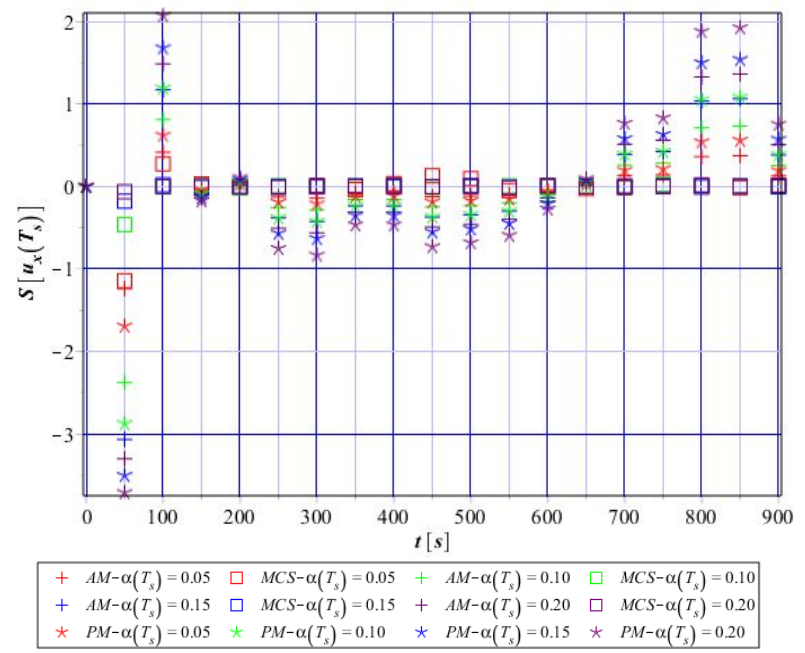

Fig. 7. Skewness of displacements.

Kurtosis of displacements (Fig. 8) takes only positive values. It means that most values of displacement are concentrated round its means. Using the Monte Carlo Simulation (MCS) and semi-analytical method (AM) we gain values close to zero. Kurtosis takes extreme twice about 50 and 800-850 second. A larger value of input coefficient of variation provides a greater value of kurtosis based on perturbation technique (PM).

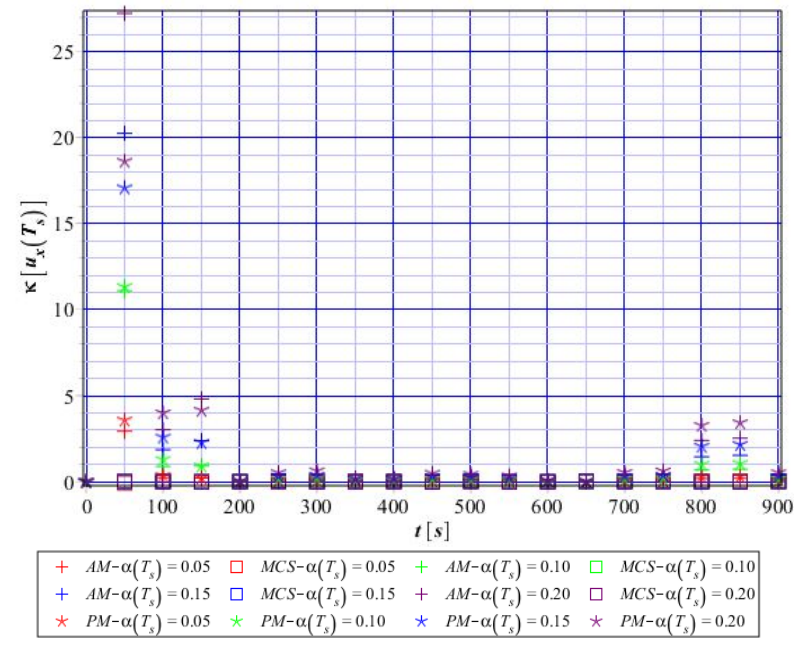

Fig. 8. Kurtosis of displacements.

\section{Conclusions}

Based on this computation we can say that the probability distribution of horizontal displacements $u_{x}$ 
under fire conditions is Gaussian if we narrow the range for the input coefficient of variation to $\alpha\left(T_{s}\right)<0.05$.

This rule refers to all three methods. Moreover, we have shown the common usage of FEM software ABAQUS and computer algebra system MAPLE, which is crucial to use Stochastic Finite Element Method (SFEM) [11]. We focused on the influence of different methods on the results of stochastic analysis. In such non-linear problems, we have to use different functions to fit the response of the structure (Fig. 4). The information about displacement distribution obtained in this study can be used in the stochastic reliability analysis of the steel structures under fire according to both First and Second Reliability Methods [3]. We show that in fire conditions methods based on the Taylor series can be an alternative for the well-known Monte Carlo Simulation (MCS).

\section{References}

1. M. Kleiber, T. D. Hien, The Stochastic Perturbation Method for Computational Mechanics (Wiley, 1992)
2. ISO 834-1, Fire-resistance tests. Elements of building construction - Part 1: General requirements

3. M. Kamiński, The Stochastic Perturbation Method for Computational Mechanics (Wiley, 2013)

4. M. Kamiński, M. Strąkowski, Arch. Civ. Mech. Eng. 13, 248-253 (2013)

5. M. Kamiński, P. Świta, Comput. Struct. 89, 12411252 (2011)

6. R. Putanowicz, J. Jaśkowiec, P. Pluciński, A. Stankiewicz, Mech. Ctrl. 32, 152-163 (2013)

7. M. Kamiński, J. Szafran, CMES: Comput. Model. Eng. Sci. 83, 143-168 (2012)

8. N. Aravas, Int. J. Numer. Meth. Eng. 24, 1395-1416 (1987)

9. O. C. Zienkiewicz, R. C. Taylor, The finite element method, Vol. I, 4th Edition (McGraw Hill, 1989)

10. EN 1993-1-2: Eurocode 3: Design of steel structures - Part 1-2: General rules - Structural fire design

11. G. Stefanou, M. Papadrakakis, Comput. Methods Appl. Mech. Eng. 193, 139-160 (2004) 